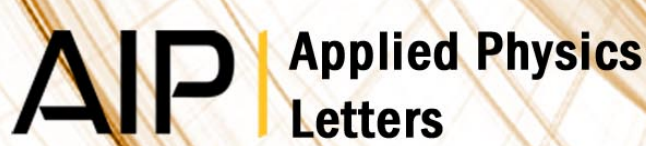

\section{Relaxation dynamics and residual strain in metamorphic AISb on GaAs}

J. M. Ripalda, A. M. Sanchez, A. G. Taboada, A. Rivera, B. Alén et al.

Citation: Appl. Phys. Lett. 100, 012103 (2012); doi: 10.1063/1.3674986

View online: http://dx.doi.org/10.1063/1.3674986

View Table of Contents: http://apl.aip.org/resource/1/APPLAB/v100/i1

Published by the American Institute of Physics.

\section{Related Articles}

Replica theory of the rigidity of structural glasses

J. Chem. Phys. 136, 214108 (2012)

Direct and quantitative evidence for buckling instability as a mechanism for roughening of polymer during plasma etching

Appl. Phys. Lett. 100, 233113 (2012)

Nonlocal elasticity based magnetic field affected vibration response of double single-walled carbon nanotube systems

J. Appl. Phys. 111, 113511 (2012)

Nano $Y^{\prime} / Y^{\prime \prime}$ composite precipitates in Alloy 718

Appl. Phys. Lett. 100, 211913 (2012)

Full-field deformation of magnetorheological elastomer under uniform magnetic field

Appl. Phys. Lett. 100, 211909 (2012)

\section{Additional information on Appl. Phys. Lett.}

Journal Homepage: http://apl.aip.org/

Journal Information: http://apl.aip.org/about/about_the_journal

Top downloads: http://apl.aip.org/features/most_downloaded

Information for Authors: http://apl.aip.org/authors

\section{ADVERTISEMENT}

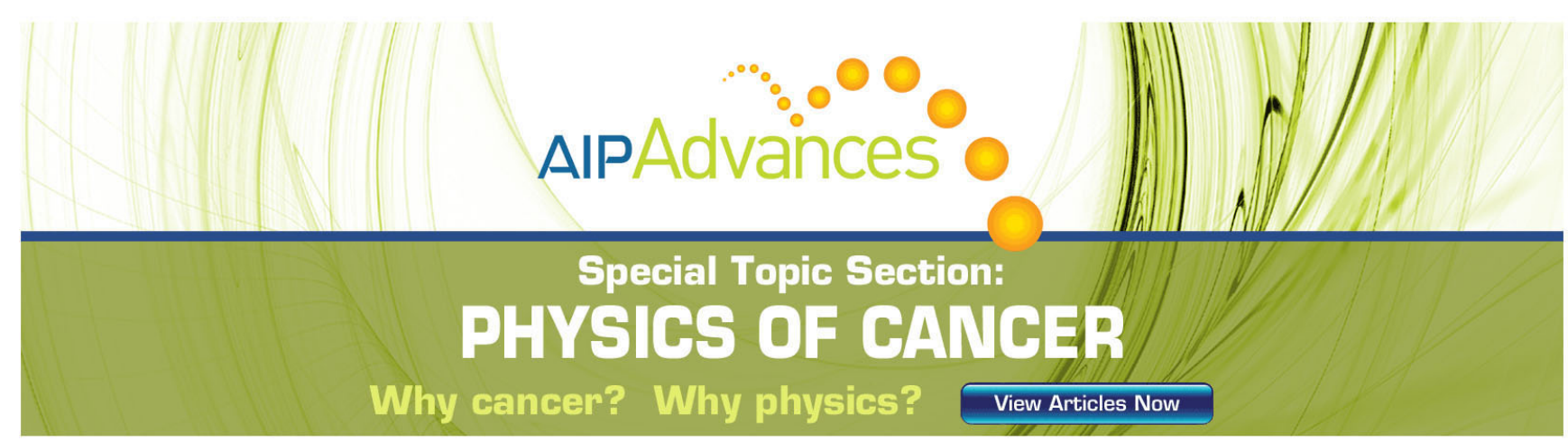




\title{
Relaxation dynamics and residual strain in metamorphic AISb on GaAs
}

\author{
J. M. Ripalda, ${ }^{1, a)}$ A. M. Sanchez, ${ }^{2}$ A. G. Taboada,${ }^{3}$ A. Rivera, ${ }^{1}$ B. Alén, ${ }^{1}$ Y. González, ${ }^{1}$ \\ L. González, ${ }^{1}$ F. Briones, ${ }^{1}$ T. J. Rotter ${ }^{4}$ and G. Balakrishnan ${ }^{4}$ \\ ${ }^{1}$ IMM-Instituto de Microelectrónica de Madrid (CNM-CSIC), Isaac Newton 8, PTM, E-28760 Tres Cantos, \\ Madrid, Spain \\ ${ }^{2}$ Physics Department, University of Warwick, Coventry CV4 7AL, United Kingdom \\ ${ }^{3}$ Laboratory for Solid State Physics, ETH Zurich, Schafmattstr. 16, CH-8093 Zurich, Switzerland \\ ${ }^{4}$ Center for High Technology Materials, University of New Mexico, Albuquerque, New Mexico 87106, USA
}

(Received 31 October 2011; accepted 12 December 2011; published online 3 January 2012)

\begin{abstract}
We have observed the evolution of the accumulated stress during heteroepitaxial growth of highly lattice mismatched AlSb on GaAs by measuring the deformation of the substrate as a function of time. High resolution transmission electron microscopy images show almost all of the plastic relaxation is accommodated by an array of $90^{\circ}$ misfit dislocations at the interface. The in-plane lattice parameter of the resulting metamorphic AlSb is slightly smaller $(0.3 \%)$ than the bulk value and perfectly matches the lattice parameter of bulk GaSb. It is, therefore, possible to grow nearly stress-free GaSb on GaAs using a metamorphic AlSb buffer layer. (c) 2012 American Institute of Physics. [doi:10.1063/1.3674986]
\end{abstract}

The growth of metamorphic III-V semiconductors on substrates of dissimilar lattice parameter is of interest for the engineering of optoelectronic devices, particularly, for those in which substrate availability would otherwise limit the applications of the material. Such is the case of the antimonides, which are very appropriate for small band gap photovoltaics or thermophotovoltaics. To increase the efficiency of tandem solar cells (typically based on Ge or GaAs substrates), it is necessary to integrate semiconductors with band gap $<1.4 \mathrm{eV}$. Metamorphic InGaAs has been integrated in GaAs based tandems, but requires the growth of thick step graded buffers of InGaP. ${ }^{1}$ Here, we present a study on the nucleation and subsequent growth of AlSb on GaAs (001) and compare our results with the more extensively studied case of $\mathrm{GaSb}$ on GaAs (Refs. 2 and 3) and the case of AlSb grown on $\mathrm{Si}^{4}{ }^{4,5}$ There is a twofold motivation to study the metamorphic growth of AlSb on GaAs rather than the more commonly studied case of GaSb on GaAs. First, the use of high band gap materials, such as AlSb, for the metamorphic interface is advantageous for integration in solar cells, where the separation of minority carriers away from defects is of utmost importance. Second, metamorphic layers are most often not $100 \%$ free of strain, but as AlSb has a slightly larger lattice parameter than GaSb $(6.1355 \AA$ vs $6.0959 \AA, 0.6 \%)$, it would, in principle, be possible to grow strain free GaSb on top of metamorphic AlSb if a small compressive strain remains in the AlSb. Metamorphic AlSb is also being actively studied for the fabrication of high electron mobility transistors ${ }^{6-8}$ and mid infrared laser sources. ${ }^{9}$

The sample was epitaxially grown on GaAs (001) by solid source molecular beam epitaxy. After oxide desorption and buffer layer growth, the sample was exposed to $\mathrm{Sb}$ at a substrate temperature $\mathrm{T}_{\mathrm{s}}$ of $600{ }^{\circ} \mathrm{C}$ until a $8 \times$ periodicity was observed in the reflection high energy electron diffraction (RHEED) pattern in the [110] azimuth, this being indicative of a Sb terminated GaAs surface. ${ }^{2}$ The substrate

a)Electronic mail: ripalda@imm.cnm.csic.es. temperature was then lowered to $500{ }^{\circ} \mathrm{C}$ and $\mathrm{AlSb}$ growth was started at $0.25 \mathrm{ML} / \mathrm{s}$ in $\mathrm{Sb}$ rich conditions $\left(2 \times 10^{-6}\right.$ mbar $\mathrm{Sb}$ beam equivalent pressure). The evolution of the accumulated stress during epitaxial growth was monitored in situ by a multi-beam optical stress sensor (MOSS). ${ }^{10}$ The substrate dimensions were $20 \times 4 \times 0.1 \mathrm{~mm}$ to maximize the deflection of the substrate due to the accumulated stress, with the longest side along the [110] direction. The deflection is measured by comparing the angle of reflection of two laser beams: one of them incident on the end that is firmly held to the substrate holder and the other incident on the free standing end of the substrate.

Transmission electron microscopy (TEM) characterization was carried out on JEOL 2100 and 2000FX microscopes, both operating at $200 \mathrm{kV}$.

The evolution of the RHEED pattern in the [11 10$]$ azimuth during AlSb epitaxy on GaAs (001) is shown in Fig. 1. The $(2 \times 8)$ reconstruction used as the initial surface for AlSb nucleation is shown in Fig. 1(a). The RHEED pattern after deposition of $0.11 \mathrm{ML}$ of AlSb on GaAs shows a spot pattern characteristic of transmission electron diffraction through the tip of 3D islands. Hao et al. have shown TEM evidence of metamorphic AlSb islands on GaAs in agreement with our observation of a RHEED pattern typical of island growth mode. ${ }^{11}$ RHEED measurements indicate a change to a lattice parameter close to that of bulk AlSb within the first ML of AlSb deposition. Such rapid change is due to the grazing incidence of the electron beam, thus the measured lattice parameter is representative of the tips of the AlSb islands which are not laterally constrained until after island coalescence. In the related case of $\mathrm{GaSb}$ on GaAs, Huang et al. have observed the coexistence of a pseudomorphic (elastically strained) wetting layer and plastically relaxed metamorphic quantum dots. ${ }^{2}$

A TEM overview of the AlSb/GaAs interface is shown in Fig. 2. The image was acquired in 220 bright field conditions, and the incident electron beam is at a $30^{\circ}$ angle relative to the plane of the interface. Most of the lattice 


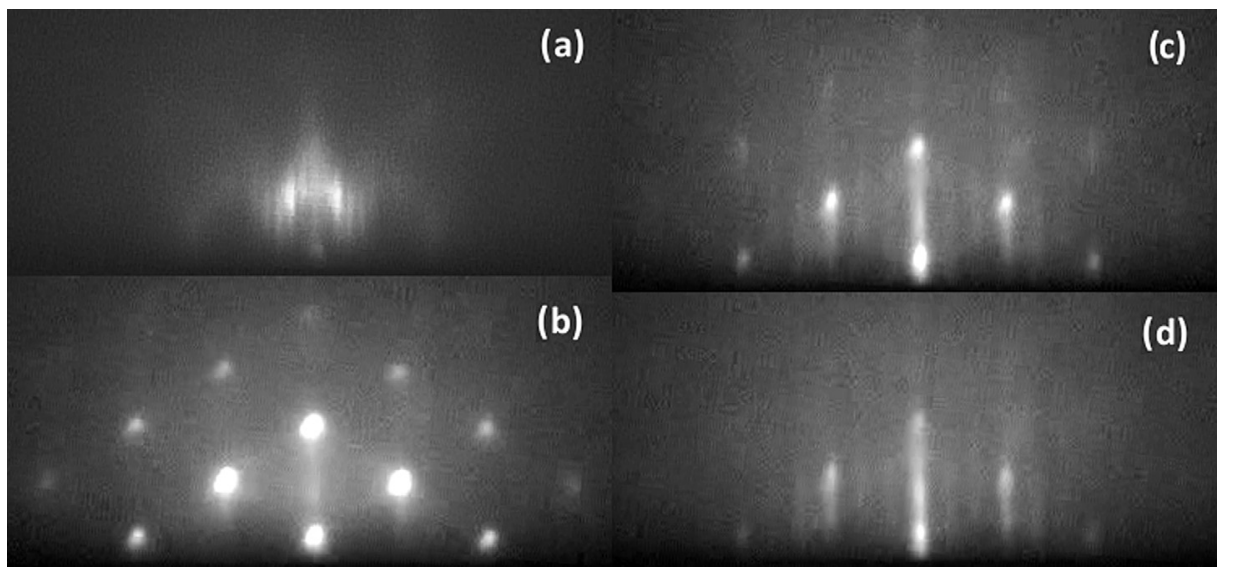

FIG. 1. RHEED pattern in the [110] azimuth at various stages of AlSb on GaAs (001) epitaxy. (a) Exposure of GaAs to antimony at $600{ }^{\circ} \mathrm{C}$ resulting in a $(2 \times 8)$ reconstruction. (b) Spot pattern indicative of 3D island nucleation. (c) Initial stage of island coalescence after deposition of 20 ML. (d) Pattern indicative of island coalescence and progress towards surface planarization after $40 \mathrm{ML}$ of AlSb on GaAs.

mismatch is relaxed by $90^{\circ}$ dislocations at the interface. The presence of planar defects and $60^{\circ}$ dislocations is also apparent in the TEM images. These can be a significant obstacle for device performance, but it has been shown that planar defects in metamorphic AlSb on GaAs can be suppressed with AlSb/GaSb super lattices. ${ }^{11}$

High resolution TEM (HRTEM) micrographs taken in a [110] projection also show a high density of interfacial misfit (IMF) dislocations (Fig. 3). Extra half planes can easily be observed at the AlSb/GaAs interface. A Burgers circuit analysis revealed that the lattice mismatch is relaxed mainly by $90^{\circ}$ dislocations with a small fraction of $60^{\circ}$ misfit dislocations. Figure 3(b) shows a map of the measured lattice parameter parallel to the interface, calculated from the lattice distortion using geometric phase methodology. ${ }^{12}$ The plot in Fig. 3(c) shows the average in-plane lattice parameter along the direction perpendicular to the interface in the rectangular area indicated in Fig. 4(b). The average in-plane AlSb lattice parameter was found to be $6.08 \pm 0.03 \AA$, slightly smaller than the lattice parameter of the bulk AlSb $(6.1355 \AA)$. Thus, only $89 \% \pm 5 \%$ of the lattice mismatch is plastically relaxed, and the growing AlSb is under compressive stress.

The accumulated stress in the epitaxial layer has been obtained from the measured substrate curvature using the following relation:

$$
\frac{-M h^{2}}{6 \mathrm{R}}=\Sigma \sigma+\Delta \tau
$$

where $R$ is the curvature radius of the substrate, $h$ is the substrate thickness, $\Sigma \sigma$ is the accumulated stress in the epitaxial layer, $M$ is the biaxial Young's modulus of the substrate, and $\Delta \tau$ is the surface tension of the substrate back surface minus the surface tension of the epitaxial front surface. In the case

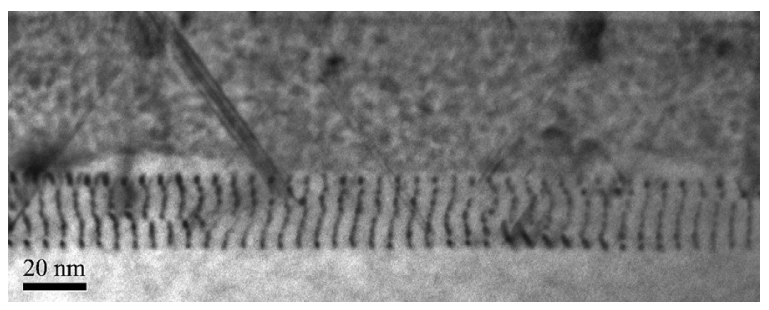

FIG. 2. Bright field 220 TEM image of the misfit dislocation array at the $\mathrm{AlSb} / \mathrm{GaAs}$ interface. of (001) substrates, $M=Y /(1-v)$, where $Y$ is Young's modulus and $v$ is the Poisson ratio of the substrate.

The evolution of the accumulated stress during epitaxial growth is shown in Fig. 4 in comparison with the expected accumulated stress curves for pseudomorphic growth and for metamorphic growth with $94.1 \%$ plastic relaxation after $2 \mathrm{ML}$ of pseudomorphic growth, the latter resulting from a linear fit to the experimental data for coverages larger than $40 \mathrm{ML}$.

The plastic/elastic relaxation ratio as determined from the MOSS stress sensor is much higher during the first ML of antimonide growth on GaAs. This is in contrast to the RHEED measurements, which seem to indicate a nearly instantaneous and complete relaxation of the lattice parameter. This apparent discrepancy is due to the fact that the RHEED measurement is predominantly probing the relaxed islands sticking out of the surface, whereas the stress measurements represent an average of the whole grown layer. This is consistent with the observation by Huang et al. of coexisting regions with and without the IMF array during the initial stages of antimonide growth. ${ }^{3}$ The TEM shows clear evidence of the IMF both in planar view and cross section, thus the results presented here are probably also relevant to other

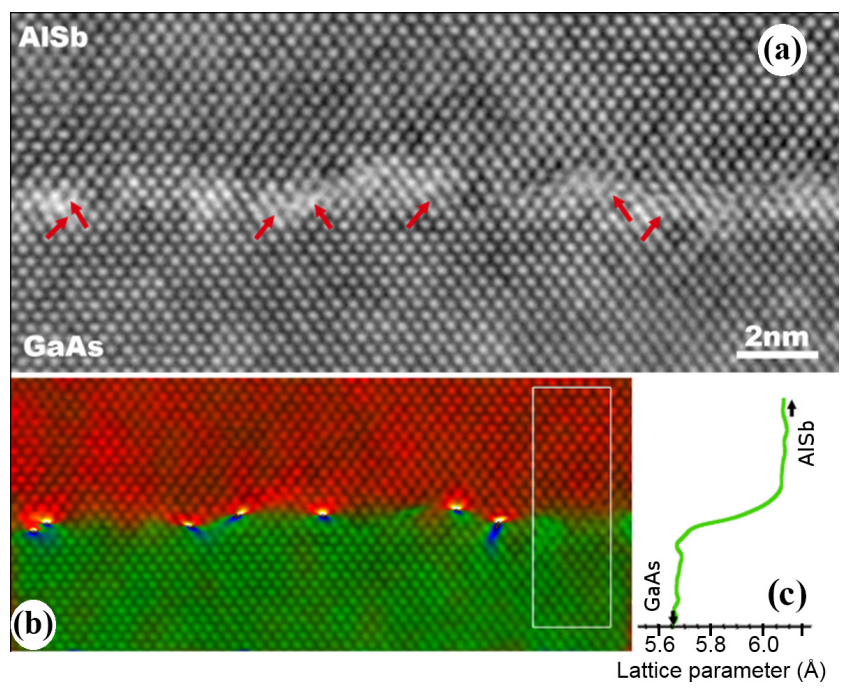

FIG. 3. (Color online) (a) High resolution TEM detail of the IMF array at the $\mathrm{AlSb} / \mathrm{GaAs}$ interface. (b) Map of the lattice distortion using geometric phase methodology. (c) Average in plane lattice parameter for the rectangular region highlighted in (b). 


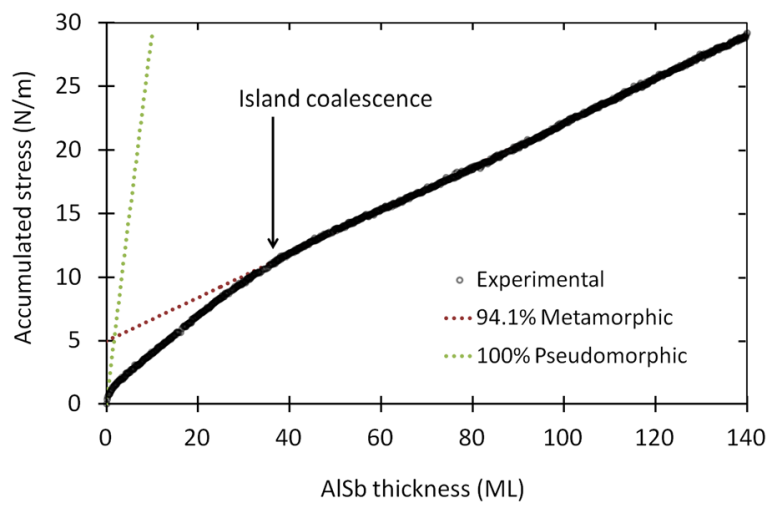

FIG. 4. (Color online) Accumulated stress as measured from the substrate deflection during growth of AlSb on GaAs.

cases of IMF metamorphic growth, such as GaAs on $\mathrm{Si}^{13}$ $\mathrm{GaSb}$ on $\mathrm{GaAs},{ }^{3}$ and $\mathrm{AlSb}$ on $\mathrm{Si}^{4}{ }^{4}$

During the first $0.76 \mathrm{ML}$ of AlSb on GaAs growth, the accumulated stress slope $(0.59 \pm 0.03 \mathrm{~N} / \mathrm{m}$ in the first 0.76 ML) corresponds approximately to the value expected for pseudomorphic growth. But changes in surface composition and reconstruction induced by $\mathrm{Sb}$ adsorption on GaAs may also contribute to changes in the measured substrate curvature due a reduction in surface tension, as can be seen in Eq. (1). Such changes of surface reconstruction and composition have been shown to induce surface tension changes of up to $1 \mathrm{~N} / \mathrm{m} .{ }^{14}$

After the first $0.8 \mathrm{ML}$, the slope rapidly decreases, suggesting the onset of stress relaxation (in this case the nucleation of the IMF dislocation array). The slope of the stress accumulation curve for coverages ranging from 10 to $20 \mathrm{ML}$ is $0.29 \pm 0.02 \mathrm{~N} / \mathrm{m} /$ per ML, approximately $10 \%$ of the value expected for pseudomorphic growth. The two contributions reducing the elastic strain are plastic relaxation and surface roughness.

A roughening of the surface can lead to a significant reduction of the slope of the accumulated stress curve, as in the case of quantum dot nucleation. ${ }^{10}$ Once the growth front reaches a planar, stationary state, the changes in the slope of the accumulated stress curve are due to plastic relaxation processes. The accumulated stress corresponding to the elastic strain deduced from HRTEM measurements $10 \mathrm{ML}$ above the interface is $6 \pm 0.3 \mathrm{~N} / \mathrm{m}$, while the accumulated stress measured in real time during growth after $10 \mathrm{ML}$ of AlSb deposition on GaAs is $4.0 \pm 0.2 \mathrm{~N} / \mathrm{m}$. This apparent discrepancy is in fact to be expected, as after $10 \mathrm{ML}$ of AlSb deposition island coalescence is still incomplete, and the growth front is still not planar, with the AlSb at the tip of the islands barely contributing to the accumulated stress. As the islands grow, these become laterally constrained as they coalesce, increasing their contribution to the accumulated stress. An example of this phenomenon was reported by Massies and Grandjean, who observed, by RHEED, oscillations in the lattice parameter of InGaAs/GaAs 2D islands corresponding to the period of monolayer completion. ${ }^{15}$ As the islands coalesce, their lattice parameter decreases, increasing the accumulated stress. Thus, there are two contributions to the slope of the accumulated stress curve: the one due to the lattice mismatch between substrate and epi-layer, and the one related to island coalescence, which is in effect due to changes of surface morphology changing the stress.

From 20 to $40 \mathrm{ML}$, there is a gradual change of slope, coinciding with the observation of island coalescence in the RHEED pattern. This change of slope in the accumulated stress curve is thus interpreted as a signature of the end of the island coalescence process. From 40 to $120 \mathrm{ML}$, the curve is linear with a slope of $0.173 \mathrm{~N} / \mathrm{m}$ per ML. This is indicative of a metamorphic AlSb lattice parameter of $6.11 \AA$, below the bulk value of $6.1355 \AA$ but in good agreement with our HRTEM measurement of the metamorphic lattice parameter for AlSb on GaAs (6.08 $\pm 0.03 \AA)$. This lattice parameter is very nearly the same as that of bulk GaSb $(6.096 \AA)$. It is therefore possible to grow nearly stress free $\mathrm{GaSb}$ on GaAs using a metamorphic AlSb buffer layer. In fact, Gotoh et al. have reported higher hole mobilities for $\mathrm{GaSb}$ grown on $\mathrm{AlSb} / \mathrm{GaAs}$ than for GaSb grown directly on GaAs. ${ }^{16}$ The AlSb layer, having a wider band gap than $\mathrm{GaSb}$, should also act as a barrier for minority carrier recombination at the interface with the substrate.

In summary, the lattice mismatch during heteroepitaxial growth of AlSb on GaAs is mostly accommodated by a misfit dislocation array confined to the interface. After deposition of $40 \mathrm{ML}$ of $\mathrm{AlSb}$ on GaAs, there is a change in the slope of the accumulated stress curve, which we interpret as an indication of the end of the island coalescence process and the consequent lateral constraining of elastically relaxed material at the tip of the islands. This hypothesis is consistent with the changes observed in the RHEED pattern. The slope of the accumulated stress curve after island coalescence is consistent with the in plane lattice parameter of metamorphic AlSb as measured by HRTEM $(6.08 \pm 0.03 \AA)$. This lateral parameter is suitable for the strain free growth of $\mathrm{GaSb}$ on GaAs substrates. Our accumulated stress measurements suggest that for this purpose, an AlSb buffer layer of at least 40 ML is required to ensure island coalescence, but no more than $140 \mathrm{ML}$ should be used to avoid the accumulation of excessive compressive stress.

We acknowledge financial support by MICINN (Grant Nos. TEC2008-06756-C03-01/03, ENE2009-14481-C02-02, CSD2006-0004, and CSD2006-0019), CAM (Grant Nos. S2009ESP-1503 and S2009/ENE-1477), and CSIC (Grant No. PIF 200950I154). A.M.S. would like to acknowledge the Science City Research Alliance and the HEFCE Strategic Development Fund for funding support. The RHEED data was analyzed with custom software tools created by Dr. David Fuster.

${ }^{1}$ J. F. Geisz, S. Kurtz, M. W. Wanlass, J. S. Ward, A. Duda, D. J. Friedman, J. M. Olson, W. E. McMahon, T. E. Moriarty, and J. T. Kiehl, Appl. Phys. Lett. 91, 023502 (2007).

${ }^{2}$ S. H. Huang, G. Balakrishnan, A. Khoshakhlagh, A. Jallipalli, L. R. Dawson, and D. L. Huffaker, Appl. Phys. Lett. 88, 131911 (2006).

${ }^{3}$ S. Huang, G. Balakrishnan, and D. Huffaker, J. Appl. Phys. 105, 103104 (2009).

${ }^{4}$ G. Balakrishnan, S. Huang, L. R. Dawson, Y.-C. Xin, P. Conlin, and D. L. Huffaker, Appl. Phys. Lett. 86, 034105 (2005).

${ }^{5}$ G. Balakrishnan, S. Huang, L. R. Dawson Y.-C. Xin, P. Conlin, and D. L. Huffaker, J. Vac. Sci. Technol. B 22(3), 1529 (2005).

${ }^{6}$ E. Lefebvre, M. Malmkvist, M. Borg, L. Desplanque, X. Wallar, G. Dambrine, S. Bollaert, and J. Grahn, IEEE Trans. Electron Devices 56, 1904 (2009). 
${ }^{7}$ H. Rodilla, T. González, G. Moschetti, J. Grahn, and J. Mateos, Semicond. Sci. Technol. 26, 025004 (2011).

${ }^{8}$ H. Rodilla, T. González, D. Pardo, and J. Mateos, J. Appl. Phys. 105, 113705 (2009)

${ }^{9}$ G. Balakrishnan, S. H. Huang, A. Khoshakhlagh, P. Hill, A. Amtout, S. Krishna, G. P. Donati, L. R. Dawson, and D. L. Huffaker, Electron. Lett. 41, 9 (2005).

${ }^{10}$ J. M. García, J. P. Silveira, and F. Briones, Appl. Phys. Lett. 77, 409 (2000).
${ }^{11}$ R. Hao, S. Deng, L. Shen, P. Yang, J. Tu, H. Liao, Y. Xu, and Z. Niu, Thin Solid Films 519, 228 (2010).

${ }^{12}$ M. J. Hÿtch, E. Snoeck, and R. Kilaas, Ultramicroscopy 74, 131 (1998).

${ }^{13}$ Y. González, L. González, and F. Briones, J. Cryst. Growth 123, 385 (1992).

${ }^{14}$ D. Fuster, M. U. González, Y. González, and L. González, Surf. Sci. 600, 23 (2006).

${ }^{15}$ J. Massies and N. Grandjean, Phys. Rev. Lett. 71, 1411 (1993).

${ }^{16}$ H. Gotoh, K. Sakamoto, S. Kuroda, and M. Kimata, Phys. Status Solidi A 75, 641 (1983). 